\title{
Characterizing Vickrey allocation rule by anonymity
}

\author{
Itai Ashlagi • Shigehiro Serizawa
}

Received: 19 June 2009 / Accepted: 2 February 2011 / Published online: 13 May 2011 (C) The Author(s) 2011. This article is published with open access at Springerlink.com

\begin{abstract}
We consider the problem of allocating finitely many units of an indivisible good among a group of agents when each agent receives at most one unit of the good and pays a non-negative price. For example, imagine that a government allocates a fixed number of licenses to private firms, or that it distributes equally divided lands to households. Anonymity in welfare is a condition of impartiality in the sense that it requires allocation rules to treat agents equally in welfare terms from the viewpoint of agents who are ignorant of their own valuations or identities. We show that the Vickrey allocation rule is the unique allocation rule satisfying strategy-proofness, anonymity in welfare, and individual rationality.
\end{abstract}

\section{Introduction}

We consider the problem of allocating finitely many units of an indivisible good among a group of agents when each agent receives at most one unit of the good and pays a non-negative price. For example, imagine that a government allocates a fixed number of licenses to private firms, or that it distributes equally divided lands to households. ${ }^{1}$

\footnotetext{
1 The vehicle ownership license system in Singapore is another example, where ownership licenses are distributed among residents through auction.
}

Two independent articles, Ashlagi (2008) and Serizawa (2006), were merged into this article.

\section{Ashlagi}

Sloan School of Management, Massachusetts Institute of Technology, Cambridge, MA 02142, USA e-mail: iashlagi@mit.edu

S. Serizawa $(\varangle)$

Institute of Social and Economic Research, Osaka University, 6-1, Mihogaoka,

Ibaraki, Osaka 567-0047, Japan

e-mail: serizawa@iser.osaka-u.ac.jp 
A number of allocation rules, including several forms of auction, have been proposed for various social purposes such as efficiency, revenue maximization, etc. For the purpose of efficiency, it is known that one rule has a remarkable feature. It is the Vickrey (allocation) rule. ${ }^{2}$ First, the Vickrey rule allocates the good to those agents who value it most highly (Efficiency). Second, it extracts true information on agents' valuations from them (Strategy-proofness). Third, it induces agents' voluntary participation (Individual rationality). Most importantly, the Vickrey rule is the unique rule satisfying these three properties. It is also known that this rule satisfies a condition of impartiality, which we call anonymity in welfare. In this paper, we characterize the Vickrey rule by focusing on this impartiality condition instead of efficiency. Our characterization emphasizes that the Vickrey rule has a remarkable feature for the purpose of impartiality as well.

An (allocation) rule is generally formulated as a function from the set of agents' valuations to the feasible set. Given a rule, as the agents' private valuations are not known to the other agents, there may be incentives for agents to misrepresent their valuations in order to manipulate the final outcomes in their favor. As a result, the actual outcomes may not constitute a socially desirable allocation relative to the agents' true valuations. If a rule is immune to such behavior, that is, if each agent's dominant strategy is to announce his true valuation, then the rule is said to be strategy-proof. In addition, a condition of individual rationality is often imposed on rules to induce agents' participation; it says that a rule never selects an allocation that makes some agent worse off than he would be if he received no good and paid nothing. It is important to know what rules satisfy efficiency, strategy-proofness, and individual rationality. The Vickrey rule does; for this rule, the agents with the $m$ highest valuations of the goods receive one unit each and pay the $(m+1)$-th valuation, while the other agents pay nothing, where $m$ is the number of the units of the indivisible good to be allocated. It is proved in the literature mentioned later that the Vickrey rule is the unique rule satisfying efficiency, strategy-proofness, and individual rationality. This result emphasizes the distinguished importance of the Vickrey rule for the purpose of efficiency in the environment where strategy-proofness and individual rationality are indispensable.

However, society members are often more concerned with impartiality than with efficiency. Anonymity in bundles is a condition of impartiality in the sense that it requires rules to treat agents equally from the viewpoint of agents who are ignorant of their own valuations or identities; it states that when the valuations of two agents are swapped, their bundles under the rule are also swapped. Anonymity in bundles is employed as a impartiality condition in many literature such as Sprumont (1991), Moulin (1994), Barberà and Jackson (1995), Serizawa (1999), etc. However, no rule satisfies anonymity in bundles in our model. A condition embodying a similar idea of impartiality is anonymity in welfare; it states that when the valuations of two agents are swapped, their welfares under the rule are also swapped. This condition is weaker than anonymity in bundle, and is satisfied by many interesting rules including the Vickrey rule even in our model. Thus, anonymity in welfare is a suitable condition of impar-

2 see Vickrey (1961) 
tiality in this article. We establish that the Vickrey rule is the unique rule satisfying strategy-proofness, anonymity in welfare, and individual rationality (Theorem 2).

We discuss other literature related to our result. Holmström (1979) analyzes models with public alternatives and money, and establishes that a rule satisfies efficiency and strategy-proofness if and only if it is a rule of the class of rules, called "Groves rules." 3 Since allocating indivisibles can be interpreted as public alternatives, his result can be applied to our model ${ }^{4}$, and imply that the Vickrey rule is the unique rule satisfying efficiency, strategy-proofness, and individual rationality. ${ }^{5}$ Recently, Chew and Serizawa (2007) characterize the Vickrey rule similarly by induction logic.

There is also a body of literature analyzing the fairness of the Vickrey rule. Most of it focuses on envy-freeness. A rule is envy-free if no agent prefers another agent's consumption bundle to his own. In a more general model with heterogeneous indivisible goods where nonquasi-linear preferences are accommodated, Svensson (1983) shows that envy-freeness of allocation implies efficiency. ${ }^{6}$ This result, together with Holmström (1979) and Chew and Serizawa (2007), implies that the Vickrey rule is the unique rule satisfying strategy-proofness, envy-freeness, and individual rationality. Many authors such as Ohseto (2006), Sakai (2008), etc., apply Svensson (1983) result to characterize the Vickrey rule. Besides, recently in problems of assigning objects, Atlamaz and Yengin (2008), and Moulin (2007) analyze variants of Vickrey rules by conditions of $k$-fairness. A rule is $k$-fair if all agents are always guaranteed $(1 / n)$-th of the $k$-th highest valuation, where $n$ is the number of agents.

In this article, we do not impose envy-freeness on rules, and so we cannot apply Svensson (1983) result. Instead, we show that strategy-proofness, anonymity in welfare, and individual rationality together imply efficiency (Proposition). This result, together with Holmström (1979), and Chew and Serizawa (2007), implies our characterization of the Vickrey rule (Theorem 2). Focusing on anonymity in welfare, our characterization emphasizes that the Vickrey rule also has a remarkable impartiality property, and it complements Holmström (1979) result, Chew and Serizawa (2007), and those of Atlamaz and Yengin (2008), and Moulin (2010).

Some authors investigate the logical relationship of anonymity with other conditions in general models. For example, Pápai (2003) results entail that under efficiency and strategy-proofness, envy-freeness implies anonymity in welfare. Yengin (2010) shows that under efficiency and strategy-proofness, equal welfare for equals is equivalent to anonymity in welfare. Our article also complements those results.

Section 2 sets up the model, defines basic notions, and states the main result. Section 3 concludes by discussing open questions. Appendix provides the proof of the

\footnotetext{
3 see Groves (1973).

4 Holmström (1979) result can be applied to more general models where there are several indivisible goods and agents consume more than one indivisible good.

5 Similar characterizations of Groves' rules in models with public alternatives have been previously established by Green and Laffont (1977) and Walker (1978). However, the characterizations of these two articles cannot be applied to allocation rules of indivisible goods because they assume that the class of admissible preferences includes preferences that are not admissible in the model of indivisible goods allocation.

6 See also Alkan et al. (1991), Maskin (1987), and Tadenuma and Thomson (1991).
} 
Proposition that strategy-proofness, anonymity in welfare, and individual rationality together imply efficiency.

\section{Model and main result}

The set of agents is $N=\{1, \ldots, n\}$. There are $m<n$ units of an indivisible good. An item allocation is a $n$-tuple $x=\left(x_{1}, \ldots, x_{n}\right) \in\{0,1\}^{n}$ such that $\sum x_{i}=m$, where for each $i \in N, x_{i}$ is the number of units of the good agent $i$ receives. ${ }^{7}$ Since $x_{i}=0$ or $x_{i}=1$ for each $i \in N$, agents can receive at most one unit of the good. We denote the set of item allocations by $X$, that is, $X=\left\{x=\left(x_{1}, \ldots, x_{n}\right) \in\{0,1\}^{n}: \sum x_{i}=m\right\}$. For each $i \in N$, we denote agent $i$ 's payment by $p_{i} \in \mathbb{R}_{+}$. We assume that payments are nonnegative. The feasible set is $Z=X \times \mathbb{R}_{+}^{n}$. An allocation is a pair $z=(x, p)=$ $\left(x_{1}, \ldots, x_{n} ; p_{1}, \ldots, p_{n}\right) \in Z$, and agent $i$ 's assignment of $z$ is $z_{i}=\left(x_{i}, p_{i}\right)$.

Each agent $i \in N$ has a quasi-linear preference (relation) $R_{i}$ on $\{0,1\} \times \mathbb{R}_{+}$; that is, there is a valuation $v_{i} \in \mathbb{R}_{+}$such that for all $\left(x_{i}, p_{i}\right) \in\{0,1\} \times \mathbb{R}_{+}$, and all $\left(\widehat{x}_{i}, \widehat{p}_{i}\right) \in\{0,1\} \times \mathbb{R}_{+},\left(x_{i}, p_{i}\right) R_{i}\left(\widehat{x}_{i}, \widehat{p}_{i}\right)$ if and only if $v_{i} \cdot x_{i}-p_{i} \geq v_{i} \cdot \widehat{x}_{i}-\widehat{p}_{i}$. Given a preference $R_{i}$, we denote the associated strict and indifference relations by $P_{i}$ and $I_{i}$ respectively. ${ }^{8}$ We denote the set of agent $i$ 's valuations by $V_{i}$. We assume $V_{i}=\mathbb{R}_{+}$.

Let $V=V_{1} \times \cdots \times V_{n}$. A valuation profile is an element $v=\left(v_{1}, \ldots, v_{n}\right) \in V$. Given $v=\left(v_{1}, \ldots, v_{n}\right) \in V, N^{\prime} \subseteq N$, and $i \in N, v_{N^{\prime}}$ denotes $\left(v_{j}\right)_{j \in N^{\prime}}$ and $v_{-i}$ denotes $\left(v_{j}\right)_{j \in N \backslash\{i\}}$. Given $v=\left(v_{1}, \ldots, v_{n}\right) \in V$ and $\widehat{v}_{i} \in V_{i},\left(\widehat{v}_{i}, v_{-i}\right)$ denotes the valuation profile $\left(v_{1}, \ldots, v_{i-1}, \widehat{v}_{i}, v_{i+1}, \ldots, v_{n}\right)$. Similarly, given $v \in V, N^{\prime} \subseteq N$, and $\widehat{v}_{N^{\prime}} \in V_{N^{\prime}} \equiv \prod_{i \in N^{\prime}} V_{i},\left(\widehat{v}_{N^{\prime}}, v_{-N^{\prime}}\right)$ denotes the valuation profile generated from $v$ by replacing the valuations of the set $N^{\prime}$ of agents by $\widehat{v}_{N^{\prime}}$.

An (allocation) rule is a function $f$ from $V$ to $Z$. Given a rule $f$ and a valuation profile $v \in V$, we write that $f(v)=(x(v), p(v))$, where $x(v)=\left(x_{1}(v), \ldots, x_{n}(v)\right)$ and $p(v)=\left(p_{1}(v), \ldots, p_{n}(v)\right)$ denote the outcome item allocation and payments of $f$ for $v$, respectively. In addition, we write $f_{i}(v)=\left(x_{i}(v), p_{i}(v)\right)$.

We introduce several conditions on rules. The first one is "efficiency." This condition states that a rule should maximize the total valuation.

Efficiency: for all $v \in V, x(v) \in \arg \max \left\{\sum_{i \in N} v_{i} \cdot x_{i}: x \in X\right\}$.

Next, we introduce impartiality conditions. The first such condition is equal bundles for equals ${ }^{9}$; it states that a rule should give the same allocation to agents with the same valuation.

Equal bundles for equals: for all $v \in V,\left[v_{i}=v_{j} \Longrightarrow f_{i}(v)=f_{j}(v)\right]$.

Equal bundles for equals is an impartiality condition that is often used in social choice theory. However, in the environment where there are fewer units to be allo-

\footnotetext{
7 We assume that all the units of the good are allocated to agents.

8 For all $z_{i}$ and $\widehat{z}_{i}$,

$$
\begin{aligned}
z_{i} P_{i} \widehat{z}_{i} & \Leftrightarrow\left[z_{i} R_{i} \widehat{z_{i}} \& \neg\left(\widehat{z_{i}} R_{i} z_{i}\right)\right], \\
z_{i} I_{i} \widehat{z}_{i} & \Leftrightarrow\left[z_{i} R_{i} \widehat{z}_{i} \& \widehat{z}_{i} R_{i} z_{i}\right] .
\end{aligned}
$$

9 This condition is also called "strong symmetry."
} 
cated than the number of agents, no rule satisfies equal bundles for equals. Thus, we introduce the following weaker condition of equal welfares for equals ${ }^{10}$; it states that a rule should give indifferent bundles to agents with the same valuation.

Equal welfares for equals: for all $v \in V,\left[v_{i}=v_{j} \Longrightarrow f_{i}(v) I_{i} f_{j}(v)\right]$.

These two conditions say nothing about agents' bundles whose valuations are not equal. Thus, we introduce "anonymity conditions." Anonymity in bundle states that when the valuations of two agents are swapped, their bundles are also swapped. This condition requires a rule to treat agents equally from the viewpoints of agents who are ignorant of their own valuations or identities.

Anonymity in bundle: for all $v \in V$, all $i \in N$, all $j \in N$, all $\widehat{v}_{i} \in V_{i}$, and all $\widehat{v}_{j} \in V_{j}$,

$$
\left[\widehat{v}_{i}=v_{j} \text { and } \widehat{v}_{j}=v_{i}\right] \Longrightarrow\left[f_{i}\left(\widehat{v}_{i}, \widehat{v}_{j}, v_{-\{i, j\}}\right)=f_{j}(v) \text { and } f_{j}\left(\widehat{v}_{i}, \widehat{v}_{j}, v_{-\{i, j\}}\right)=f_{i}(v)\right] \text {. }
$$

Note that anonymity in bundle implies equal bundles for equals, and that in our environment, no rule satisfies anonymity in bundle. Thus, we introduce a weaker condition of anonymity in welfare; it states that when the valuations of two agents are swapped, their welfare positions are also swapped. ${ }^{11}$

Anonymity in welfare: for all $v \in V$, all $i \in N$, all $j \in N$, all $\widehat{v}_{i} \in V_{i}$, and all $\widehat{v}_{j} \in V_{j}$,

$$
\left[\widehat{v}_{i}=v_{j} \text { and } \widehat{v}_{j}=v_{i}\right] \Longrightarrow\left[\widehat{v}_{i} \cdot \widehat{x}_{i}-\widehat{p}_{i}=v_{j} \cdot x_{j}-p_{j} \text { and } \widehat{v}_{j} \cdot \widehat{x}_{j}-\widehat{p}_{j}=v_{i} \cdot x_{i}-p_{i}\right] \text {, }
$$

where $x_{k}=x_{k}(v), p_{k}=p_{k}(v), \widehat{x}_{k}=x_{k}\left(\widehat{v}_{i}, \widehat{v}_{j}, v_{-\{i, j\}}\right)$ and $\widehat{p}_{k}=p_{k}\left(\widehat{v}_{i}, \widehat{v}_{j}, v_{-\{i, j\}}\right)$ for $k=i, j$.

Note that anonymity in welfare implies equal welfares for equals . In Sect. 3, we employ this implication repeatedly to prove the Proposition below.

Envy-freeness requires that no agent should prefer another agent's bundle to his own. In contrast to the above anonymity conditions, this condition compares agents' welfare from the viewpoints of agents whose identities and valuations are specified.

Envy-freeness: for all $v \in V$, all $i \in N$, and all $j \in N, f_{i}(v) R_{i} f_{j}(v)$.

The next requirement, strategy-proofness, is that for each agent, revealing his true valuation should be a dominant strategy in the direct revelation game. Since the dominant strategy equilibrium is a strong equilibrium concept, strategy-proofness gives agents strong incentives to represent their true valuations.

Strategy-proofness: for all $v \in V$, all $i \in N$, and all $\widehat{v}_{i} \in V_{i}, f_{i}(v) R_{i} f_{i}\left(\widehat{v}_{i}, v_{-i}\right)$.

Individual rationality induces agents to participate voluntarily by guaranteeing that a rule never assigns an outcome that makes some agent worse off than he would be at his status quo $(0,0)$.

Individual rationality: for all $v \in V$ and all $i \in N, f_{i}(v) R_{i}(0,0)$.

\footnotetext{
10 This condition is also called "symmetry."

11 An alternative definition of anonymity in welfare is that when the valuations of a group of agents are permutated, their welfare positions are permutated in the same way. However, since any permutation can be written as a product of simple transpositions, the two definitions of anonymity in welfare of are equivalent.
} 
Given a valuation profile $v \in V$, we rank agents' valuations, and denote agent $i$ 's rank by $r(v, i)$. That is, if $v_{i}$ is the highest in $\left\{v_{1}, \ldots, v_{n}\right\}, r(v, i)=1$; if it is the second highest, $r(v, i)=2$; and so on. When several agents have the same valuation, ties are broken in a prespecified way.

One example of tie-breaker is to use their indices in increasing order. For instance, if a valuation profile is $v=\left(v_{1}, v_{2}, v_{3}, v_{4}\right)=(5,4,7,5)$, then $r(v, 1)=2, r(v, 2)=$ $4, i(v, 3)=1$, and $r(v, 4)=3$. Ranking agents with the same valuation according to their indices in decreasing order is another example. Thus, a ranking function $r(\cdot, \cdot)$ is associated with a certain tie-breaker. It is possible to construct ranking functions with more complex tie-breakers such as examples below.

Example 1 Let $n=2$. The ranking function $r(\cdot, \cdot)$ is such that $r(v, 1)=1$ if $v_{1}=$ $v_{2} \geq 4$, but $r(v, 1)=2$ if $v_{1}=v_{2}<4$. In this ranking function, when the two agents have the same valuation, which agent is ranked first depends on the level of the common valuation.

Example 2 Let $n=3$. The ranking function $r(\cdot, \cdot)$ is such that if $v_{1}=v_{2} \geq v_{3}$, then $r(v, 1)=1$ and $r(v, 2)=2$; if $v_{1}=v_{2}<v_{3}$, then $r(v, 1)=3$ and $r(v, 2)=2$. In this ranking function, when agents 1 and 2 have the same valuation, which of agent 1 or 2 is ranked higher depends on agent 3's valuation.

Given a valuation profile $v \in V$ and a ranking function $r(\cdot, \cdot)$, we denote the inverse of $r(v, \cdot)$ by $i(v, \cdot)$, that is, $i(v, k)$ is the agent whose valuation is the $k$-th highest. For instance, if a valuation profile is $v=\left(v_{1}, v_{2}, v_{3}, v_{4}\right)=(5,4,7,5)$, and $r(v, 1)=2, r(v, 2)=4, r(v, 3)=1$, and $r(v, 4)=3$, then $i(v, 1)=3, i(v, 2)=$ $1, i(v, 3)=4$, and $i(v, 4)=2$.

Under the Vickrey rule defined below, agents with the $m$ highest valuations receive the goods and pay the $(m+1)$-th highest valuation, $v_{i(v, m+1)}$, and the other agents pay nothing. For instance, suppose that ties are broken according to agents' indices in increasing order. Consider the case in which $n=3, m=1$, and a valuation profile is $\left(v_{1}, v_{2}, v_{3}\right)=(1,1,0)$. Then, agents 1 receives the good, and since the 2 -nd highest valuation $v_{i(v, 2)}$ is $v_{2}=1$, agents 1 pays 1 . Or consider the case in which $n=6, m=3$, and a valuation profile is $\left(v_{1}, v_{2}, v_{3}, v_{4}, v_{5}, v_{6}\right)=(5,3,7,5,2,3)$. Then, agents 1,3 , and 4 receive the good, and since the 4 -th highest valuation $v_{i}(v, 4)$ is $v_{2}=3$, agents 1,3 and 4 pay 3 .

Definition A Vickrey (allocation) rule is a rule $f^{*}=\left(x^{*}(\cdot), p^{*}(\cdot)\right)$ such that:

for all $v \in V$ and all $i \in N, \quad\left(x_{i}^{*}(v), p_{i}^{*}(v)\right)= \begin{cases}\left(1, v_{i(v, m+1)}\right) & \text { if } r(v, i) \leq m, \text { and } \\ (0,0) & \text { otherwise. }\end{cases}$

Since the Vickrey rules are accompanied by ranking functions, and since ranking functions are not unique, the Vickrey rules are not unique. In other words, there are as many Vickrey rules as ways of tie-breaking. However, unless several agents have the $m$-th highest valuation, regardless of ranking function, all the Vickrey rules give the same allocation. Furthermore, as Remark below states, all the Vickrey rules are welfare-equivalent. Therefore, we treat Vickrey rules as if they were unique. 
Remark Let $f^{*}$ and $\widehat{f}^{*}$ be Vickrey rules. Then:

(i) $\sum_{i \in N} x_{i}^{*}(v)=\sum_{i \in N} \widehat{x}_{i}^{*}(v)$ for any $v \in V$,

(ii) $v_{i} \cdot x_{i}^{*}(v)-p_{i}^{*}(v)=v_{i} \cdot \widehat{x}_{i}^{*}(v)-\widehat{p}_{i}^{*}(v)$ for any $v \in V$ and any $i \in N$,

where $x_{i}^{*}(v)$ and $\widehat{x}_{i}^{*}(v)$ are, respectively, agent $i$ 's item allocations under $f^{*}$ and $\widehat{f}^{*}$ for $v$, and $p_{i}^{*}(v)$ and $\widehat{p}_{i}^{*}(v)$ are, respectively, his payments under $f^{*}$ and $\widehat{f}^{*}$ for $v$.

By its definition, the Vickrey rule satisfies efficiency. It is straightforward that the Vickrey rule satisfies strategy-proofness and individual rationality. Theorem 1 below states that the Vickrey rule is the unique rule satisfying those three conditions.

Theorem 1 A rule satisfies strategy-proofness, efficiency, and individual rationality if and only if it is the Vickrey rule.

Theorem follows from Holmström (1979). The proof given below is a simplified version of the proof given by Chew and Serizawa (2007).

Proof We only show that if a rule satisfies strategy-proofness, efficiency, and individual rationality, then it is the Vickrey rule. Let a rule $f$ satisfy strategy-proofness, efficiency and individual rationality. Suppose that $f$ is not the Vickrey rule.

Since $f$ satisfies efficiency and individual rationality, there are $v \in V$ and $i \in N$ such that $v_{i} \geq v_{i(v, m)}, x_{i}(v)=1$ and $p_{i}(v) \neq v_{i(v, m+1)}$. Since $f$ satisfies individual rationality, $p_{i}(v) \leq v_{i}$. Thus, $v_{i(v, m+1)}<p_{i}(v) \leq v_{i}$, or $p_{i}(v)<v_{i(v, m+1)}$.

First, consider the case where $v_{i(v, m+1)}<p_{i}(v) \leq v_{i}$. Let $\widehat{v}_{i} \in\left(v_{i}(v, m+1), p_{i}(v)\right)$. Then, since $f$ satisfies efficiency and individual rationality, $x_{i}\left(\widehat{v}_{i}, v_{-i}\right)=1$ and $p_{i}\left(\widehat{v}_{i}, v_{-i}\right) \leq \widehat{v}_{i}<p_{i}(v)$. Thus, $v_{i} \cdot x_{i}\left(\widehat{v}_{i}, v_{-i}\right)-p_{i}\left(\widehat{v}_{i}, v_{-i}\right)>v_{i} \cdot x_{i}(v)-p_{i}(v)$, contradicting strategy-proofness.

Next, consider the case where $p_{i}(v)<v_{i(v, m+1)}$. Let $\widehat{v}_{i} \in\left(p_{i}(v), v_{i(v, m+1)}\right)$. Then, since $f$ satisfies efficiency and individual rationality, $x_{i}\left(\widehat{v}_{i}, v_{-i}\right)=0$ and $p_{i}\left(\widehat{v}_{i}, v_{-i}\right)=0$. Thus, $\widehat{v}_{i} \cdot x_{i}(v)-p_{i}(v)>\widehat{v}_{i} \cdot x_{i}\left(\widehat{v}_{i}, v_{-i}\right)-p_{i}\left(\widehat{v}_{i}, v_{-i}\right)$, contradicting strategy-proofness.

Hence, $f$ is the Vickrey rule.

No rule satisfies anonymity in bundle in our model, but all the Vickrey rules satisfy anonymity in welfare. The Proposition below says that the condition of anonymity in welfare, together with strategy-proofness and individual rationality, implies efficiency. We prove this Proposition in Appendix.

Proposition If a rule satisfies strategy-proofness, anonymity in welfare, and individual rationality, it also satisfies efficiency.

As Svensson (1983) shows, envy-freeness alone implies efficiency . As Example 3 illustrates, anonymity in welfare alone does not imply efficiency.

Example 3 Let $m=1$ and $n=2$. Let $f$ be the rule such that if $v_{1} \leq v_{2}, f(v)=$ $\left((1,0) ;\left(v_{2}, 0\right)\right)$, and if $v_{1}>v_{2}, f(v)=\left((0,1) ;\left(0, v_{1}\right)\right)$. Then, $f$ satisfies anonymity in welfare but not efficiency. The rule $f$ also violates strategy-proofness and individual rationality. 
As we mentioned above, the Vickrey rule satisfies strategy-proofness, individual rationality and anonymity in welfare. Thus, Theorem 1 and the Proposition together imply Theorem 2 below. Theorem 2 states that the Vickrey rule is the unique rule satisfying those three conditions.

Theorem 2 A rule satisfies strategy-proofness, anonymity in welfare, and individual rationality if and only if it is the Vickrey rule.

The three examples below illustrate that the three axioms in Theorem 2 are independent.

Example 4 Let $m=1$ and $n=2$. Let $f$ be the rule such that if $v_{1} \geq 1, f(v)=$ $((1,0) ;(1,0))$, and if $v_{1}<1, f(v)=((0,1) ;(0,0))$. Then, $f$ satisfies strategyproofness and individual rationality, but not anonymity in welfare.

Example 5 Let $m=1$ and $n=2$. Let $f$ be the rule such that if $v_{1} \geq v_{2}, f(v)=$ $\left((1,0) ;\left(v_{1}, 0\right)\right)$, and if $v_{1}<v_{2}, f(v)=\left((0,1) ;\left(0, v_{2}\right)\right)$. Then, $f$ satisfies anonymity in welfare, and individual rationality, but not strategy-proofness.

Example 6 Let $m=1$ and $n=2$. Let $f$ be the rule such that if $v_{1} \geq v_{2}, f(v)=$ $\left((1,0) ;\left(2 v_{2}, v_{1}\right)\right)$, and if $v_{1}<v_{2}, f(v)=\left((0,1) ;\left(v_{2}, 2 v_{1}\right)\right)$. Then, $f$ satisfies strategy-proofness, and anonymity in welfare, but not individual rationality.

\section{Concluding remarks}

In this article, we considered the problem of allocating finitely many units of an indivisible good when each agent receives at most one unit of the good and pays a non-negative price. We established that strategy-proofness, anonymity in welfare, and individual rationality together imply efficiency, and that the Vickrey rule is the unique rule satisfying strategy-proofness, anonymity in welfare, and individual rationality. We close this article by mentioning open questions we left.

As we discussed in Introduction, Svensson (1983) showed that envy-freeness of allocation implies efficiency in a more general model with heterogeneous indivisible goods where even nonquasi-linear preferences are accommodated. The results of Holmström (1979) and Chew and Serizawa (2007) can be applied to general models with heterogeneous indivisible goods where agents may consume more than one indivisible good as long as preferences are quasi-linear. ${ }^{12}$ That is, the Vickrey rule is the unique rule satisfying strategy-proofness, efficiency, and individual rationality in such general models. We conjecture that (i) strategy-proofness, anonymity in welfare, and individual rationality together also imply efficiency in general models, and that (ii) the Vickrey rule is also the unique rule satisfying strategy-proofness, anonymity in welfare, and individual rationality in general models.

Hashimoto and Saitoh (2009) recently studied a queuing model in which preferences are quasi-linear, and are represented by constant unit waiting cost. They applied

\footnotetext{
12 Saitoh and Serizawa (2008) showed a parallel result in the model with homogenous indivisible goods and nonquasi-linear preferences. See also Roth and Sotomayor (1990)
} 
the proof techniques of our article to show that strategy-proofness and anonymity in welfare imply efficiency. Since positions in queue can be interpreted as heterogeneous indivisibles, their model is a model of heterogeneous indivisibles. Although their generalization is limited in the sense that preferences are represented by single parameters, Hashimoto and Saitoh (2009) result suggests that the techniques we developed in our article will be useful in generalized models if the techniques are modified suitably. However, such modifications need considerable amount of effort, and so we leave them in this article although we believe that they are interesting questions in future research.

As Pápai (2003) showed, when agents may consume more than one indivisible good and valuations are not restricted, no rule in the class of Groves (including the Vickrey rule) satisfies envy-freeness. ${ }^{13}$ On the other hand, the Vickrey rule satisfies anonymity in welfare even when agents may consume more than one indivisible good. Thus, the conjecture (ii) above will contrast anonymity in welfare with envy-freeness if it is established.

\section{Appendix: proof of proposition}

In this appendix, we prove the Proposition stated in Sect. 2. First, we state and prove several lemmas as preliminary results.

Lemma 1 Let $f$ be an individually rational rule. For all $v \in V$ and all $i \in N$, if $x_{i}(v)=0$, then $p_{i}(v)=0$.

Lemma 1 directly follows from individual rationality. Thus, we omit its proof.

Lemma 2 Let $f$ be a strategy-proof rule. For all $v \in V$, all $i \in N$, and all $\widehat{v}_{i} \in V_{i}$, if $x_{i}\left(\widehat{v}_{i}, v_{-i}\right)=x_{i}(v)$, then $p_{i}\left(\widehat{v}_{i}, v_{-i}\right)=p_{i}(v)$.

Proof Let $v \in V, i \in N$, and $\widehat{v}_{i} \in V_{i}$ be such that $x_{i}\left(\widehat{v}_{i}, v_{-i}\right)=x_{i}(v)$. If $p_{i}\left(\widehat{v}_{i}, v_{-i}\right)>$ $p_{i}(v), f_{i}(v) \widehat{P}_{i} f_{i}\left(\widehat{v}_{i}, v_{-i}\right)$, and if $p_{i}\left(\widehat{v}_{i}, v_{-i}\right)<p_{i}(v), f_{i}\left(\widehat{v}_{i}, v_{-i}\right) P_{i} f_{i}(v)$. Both cases contradict strategy-proofness. Thus, $p_{i}\left(\widehat{v}_{i}, v_{-i}\right)=p_{i}(v)$.

Lemma 3 Let $f$ be a strategy-proof and individually rational rule.

(a) For all $v \in V$, all $i \in N$, and all $\widehat{v}_{i} \in V_{i}$, if $x_{i}(v)=1$ and $\widehat{v}_{i}>v_{i}$, then $x_{i}\left(\widehat{v}_{i}, v_{-i}\right)=1$ and $p_{i}\left(\widehat{v}_{i}, v_{-i}\right)=p_{i}(v)$.

(b) For all $v \in V$, all $i \in N$, and all $\widehat{v}_{i} \in V_{i}$, if $x_{i}(v)=0$ and $\widehat{v}_{i}<v_{i}$, then $x_{i}\left(\widehat{v}_{i}, v_{-i}\right)=0$ and $p_{i}\left(\widehat{v}_{i}, v_{-i}\right)=0$.

Proof of a: Let $v \in V, i \in N$, and $\widehat{v}_{i} \in V_{i}$ be such that $x_{i}(v)=1$ and $\widehat{v}_{i}>v_{i}$. Suppose by contradiction that $x_{i}\left(\widehat{v}_{i}, v_{-i}\right)=0$. Then, by Lemma $1, p_{i}\left(\widehat{v}_{i}, v_{-i}\right)=0$. By individual rationality, $p_{i}(v) \leq v_{i}<\widehat{v}_{i}$. Thus, $\widehat{v}_{i} \cdot x_{i}(v)-p_{i}(v)>0=\widehat{v}_{i} \cdot x_{i}\left(\widehat{v}_{i}, v_{-i}\right)$ $-p_{i}\left(\widehat{v}_{i}, v_{-i}\right)$. This contradicts strategy-proofness. Therefore, $x_{i}\left(\widehat{v}_{i}, v_{-i}\right)=1=x_{i}(v)$, and by Lemma $2, p_{i}\left(\widehat{v}_{i}, v_{-i}\right)=p_{i}(v)$.

$\overline{13}$ This negative result holds when preferences are not restricted unless they are quasi-linear. 
Proof of b: Let $v \in V, i \in N$, and $\widehat{v}_{i} \in V_{i}$ be such that $x_{i}(v)=0$ and $\widehat{v}_{i}<v_{i}$. Suppose by contradiction that $x_{i}\left(\widehat{v}_{i}, v_{-i}\right)=1$. By Lemma $1, p_{i}(v)=$ 0 . By individual rationality, $p_{i}\left(\widehat{v}_{i}, v_{-i}\right) \leq \widehat{v}_{i}<v_{i}$. Thus, $v_{i} \cdot x_{i}\left(\widehat{v}_{i}, v_{-i}\right)$ $-p_{i}\left(\widehat{v}_{i}, v_{-i}\right)>0=v_{i} \cdot x_{i}(v)-p_{i}(v)$. This contradicts strategy-proofness. Therefore, $x_{i}\left(\widehat{v}_{i}, v_{-i}\right)=0$, and by Lemma $1, p_{i}\left(\widehat{v}_{i}, v_{-i}\right)=0$.

Lemma 4 Let $f$ be a rule satisfying strategy-proofness, anonymity in welfare, and individual rationality.

(a) Let $v \in V, i \in N, \widehat{v}_{i} \in V_{i}, v_{0}>0$, and $N^{\prime} \subseteq N$ be such that $x_{i}(v)=1, v_{i}<$ $v_{0}=\widehat{v}_{i}$, and $v_{j}=v_{0}$ for all $j \in N^{\prime}$. Then, $x_{i}\left(\widehat{v}_{i}, v_{-i}\right)=x_{j}\left(\widehat{v}_{i}, v_{-i}\right)=1$ for all $j \in N^{\prime}$.

(b) Let $v \in V, i \in N, \widehat{v}_{i} \in V_{i}, v_{0} \geq 0$, and $N^{\prime} \subseteq N$ be such that $x_{i}(v)=0, v_{i}>$ $v_{0}=\widehat{v}_{i}$, and $v_{j}=v_{0}$ for all $j \in N^{\prime}$. Then, $x_{i}\left(\widehat{v}_{i}, v_{-i}\right)=x_{j}\left(\widehat{v}_{i}, v_{-i}\right)=0$ for all $j \in N^{\prime}$.

Proof of a: By Lemma 3(a), since $x_{i}(v)=1$ and $v_{i}<\widehat{v}_{i}$, we have $x_{i}\left(\widehat{v}_{i}, v_{-i}\right)=1$ and $p_{i}\left(\widehat{v}_{i}, v_{-i}\right)=p_{i}(v) \leq v_{i}<v_{0}$. Thus, since $v_{j}=v_{0}=\widehat{v}_{i}$ for all $j \in N^{\prime}$, it follows from anonymity in welfare that $v_{j} \cdot x_{j}\left(\widehat{v}_{i}, v_{-i}\right)-p_{j}\left(\widehat{v}_{i}, v_{-i}\right)=\widehat{v}_{i} \cdot x_{i}\left(\widehat{v}_{i}, v_{-i}\right)$ $-p_{i}\left(\widehat{v}_{i}, v_{-i}\right) \geq v_{0}-p_{i}(v)>0$ for all $j \in N^{\prime}$. Therefore, by Lemma $1, x_{j}\left(\widehat{v}_{i}, v_{-i}\right)=1$ for all $j \in N^{\prime}$.

Proof of b: By Lemma 1, since $x_{i}(v)=0$, we have $p_{i}(v)=0$. By Lemma 3(b), since $x_{i}(v)=0$, and $\widehat{v}_{i}<v_{i}$, we have $x_{i}\left(\widehat{v}_{i}, v_{-i}\right)=0$, and so by Lemma $1, p_{i}\left(\widehat{v}_{i}, v_{-i}\right)=0$. Thus, since $v_{j}=v_{0}=\widehat{v}_{i}$ for all $j \in N^{\prime}$, it follows from anonymity in welfare that $v_{j} \cdot x_{j}\left(\widehat{v}_{i}, v_{-i}\right)-p_{j}\left(\widehat{v}_{i}, v_{-i}\right)=\widehat{v}_{i} \cdot x_{i}\left(\widehat{v}_{i}, v_{-i}\right)-p_{i}\left(\widehat{v}_{i}, v_{-i}\right)=0$ for all $j \in N^{\prime}$. Therefore, for all $j \in N^{\prime}$, if $x_{j}\left(\widehat{v}_{i}, v_{-i}\right)=1, p_{j}\left(\widehat{v}_{i}, v_{-i}\right)=v_{0}$.

Since $x_{i}\left(\widehat{v}_{i}, v_{-i}\right)=0$, suppose that there is $j \in N^{\prime}$ such that $x_{j}\left(\widehat{v}_{i}, v_{-i}\right)=1$. Let $\widehat{v}_{j}=v_{i}$. Then, since $v_{i}>v_{0}=v_{j}$, Lemma 3(a) implies that $x_{j}\left(\widehat{v}_{i}, \widehat{v}_{j}, v_{-\{i, j\}}\right)=$ $1, p_{j}\left(\widehat{v}_{i}, \widehat{v}_{j}, v_{-\{i, j\}}\right)=p_{j}\left(\widehat{v}_{i}, v_{-i}\right)=v_{0}<v_{i}=\widehat{v}_{j}$, and $\widehat{v}_{j} \cdot x_{j}\left(\widehat{v}_{i}, \widehat{v}_{j}, v_{-\{i, j\}}\right)$ $-p_{j}\left(\widehat{v}_{i}, \widehat{v}_{j}, v_{-\{i, j\}}\right)=\widehat{v}_{j}-v_{0}>0$. Since $v_{i} \cdot x_{i}(v)-p_{i}(v)=0, \widehat{v}_{i}=v_{0}=v_{j}$, and $\widehat{v}_{j}=v_{i}$, this contradicts anonymity in welfare. Hence, for all $j \in N^{\prime}, x_{j}\left(\widehat{v}_{i}, v_{-i}\right)=$ 0 .

Now we prove the Proposition.

Let $f$ be a rule satisfying strategy-proofness, anonymity in welfare, and individual rationality. Let $v \in V$. We show that $f(v)$ is efficient for $v$. Without loss of generality, assume $v_{1} \geq v_{2} \geq \cdots \geq v_{m}=\cdots=v_{n^{\prime}}>v_{n^{\prime}+1} \geq v_{n^{\prime}+2} \geq \cdots \geq v_{n},{ }^{14}$ and let $\bar{v}=v_{m}$. Note that for all $i \in N, v_{i}>\bar{v}$ implies $i<m$, and that $v_{j}<\bar{v}$ implies $j>n^{\prime}$. Therefore, $f(v)$ is efficient for $v$ if and only if

(A) for all $i \in N, v_{i}>\bar{v}$ implies $x_{i}(v)=1$, and

(B) for all $i \in N, x_{i}(v)=1$ implies $i \leq n^{\prime}$.

Thus, if $f(v)$ is not efficient for $v$, then $(\mathrm{A})$ or $(\mathrm{B})$ is violated.

\footnotetext{
$\overline{14} n^{\prime}$ may be equal to $m$ or $n$. In the case where $n^{\prime}=m, v_{1} \geq v_{2} \geq \cdots \geq v_{m}>v_{m+1} \geq v_{m+2} \geq \cdots \geq v_{n}$. In the case where $n^{\prime}=n, v_{1} \geq v_{2} \geq \cdots \geq v_{m}=v_{m+1}=\cdots=v_{n}$.
} 
If (B) is violated, that is, if there is an agent $i \in N$ such that $x_{i}(v)=1$ and $i>n^{\prime}$, then we raise his valuation to $\widehat{v}_{i}=\bar{v}$, and by Lemma 4(a), we obtain that

$$
x_{i}\left(\widehat{v}_{i}, v_{-i}\right)=x_{m}\left(\widehat{v}_{i}, v_{-i}\right)=x_{m+1}\left(\widehat{v}_{i}, v_{-i}\right)=\cdots=x_{n^{\prime}}\left(\widehat{v}_{i}, v_{-i}\right)=1 \text {. }
$$

Since $i>n^{\prime}$ and there are only $m$ units of the goods, it follows that there is an agent $j<m$ such that $x_{j}\left(\widehat{v}_{i}, v_{-i}\right)=0 . j<m$ implies $v_{j} \geq \bar{v}$. If $v_{j}=\bar{v}$, Lemma 4(a) implies $x_{j}\left(\widehat{v}_{i}, v_{-i}\right)=1$, contradicting $x_{j}\left(\widehat{v}_{i}, v_{-i}\right)=0$. Thus, $v_{j}>\bar{v}$. For $j \in N$, (A) is violated. Thus, we assume that (A) is violated and derive a contradiction.

Since (A) is violated, there is an agent $i$ such that $v_{i}>\bar{v}$ and $x_{i}(v)=0 . v_{i}>\bar{v}$ implies $i<m$. We lower his valuation to $\widehat{v}_{i}=\bar{v}$, and by Lemma 4(b), we obtain

$$
x_{i}\left(\widehat{v}_{i}, v_{-i}\right)=x_{m}\left(\widehat{v}_{i}, v_{-i}\right)=x_{m+1}\left(\widehat{v}_{i}, v_{-i}\right)=\cdots=x_{n^{\prime}}\left(\widehat{v}_{i}, v_{-i}\right)=0 .
$$

Since $i<m$ and the supply is $m$, there is an agent $j$ such that $j>n^{\prime}$, and $x_{j}\left(\widehat{v}_{i}, v_{-i}\right)=$ 1. $j>n^{\prime}$ implies $v_{j}<\bar{v}$.

We raise the valuation of $j$ to $\widehat{v}_{j}=\bar{v}$, and by Lemma 4(a), we obtain

$$
\begin{gathered}
x_{j}\left(\widehat{v}_{i}, \widehat{v}_{j}, v_{-\{i, j\}}\right)=x_{i}\left(\widehat{v}_{i}, \widehat{v}_{j}, v_{-\{i, j\}}\right)=x_{m}\left(\widehat{v}_{i}, \widehat{v}_{j}, v_{-\{i, j\}}\right) \\
=x_{m+1}\left(\widehat{v}_{i}, \widehat{v}_{j}, v_{-\{i, j\}}\right)=\cdots=x_{n^{\prime}}\left(\widehat{v}_{i}, \widehat{v}_{j}, v_{-\{i, j\}}\right)=1 .
\end{gathered}
$$

Since there are only $m$ units of the goods, it follows that there is an agent $i^{\prime}<m$ such that $i^{\prime} \neq i$, and $x_{i^{\prime}}\left(\widehat{v}_{i}, \widehat{v}_{j}, v_{-\{i, j\}}\right)=0 . i^{\prime}<m$ implies $v_{i^{\prime}} \geq \bar{v}$. If $v_{i^{\prime}}=\bar{v}$, Lemma 4(a) implies $x_{i^{\prime}}\left(\widehat{v}_{i}, \widehat{v}_{j}, v_{-\{i, j\}}\right)=1$, contradicting $x_{i^{\prime}}\left(\widehat{v}_{i}, \widehat{v}_{j}, v_{-\{i, j\}}\right)=0$. Thus, $v_{i^{\prime}}>\bar{v}$.

Again, we lower the valuation of $i^{\prime}$ to $\widehat{v}_{i^{\prime}}=\bar{v}$ and by Lemma 4(b), we obtain

$$
\begin{aligned}
& x_{i^{\prime}}\left(\widehat{v}_{i}, \widehat{v}_{i^{\prime}}, \widehat{v}_{j}, v_{-\left\{i, i^{\prime}, j\right\}}\right)=x_{i}\left(\widehat{v}_{i}, \widehat{v}_{i^{\prime}}, \widehat{v}_{j}, v_{-\left\{i, i^{\prime}, j\right\}}\right)=x_{j}\left(\widehat{v}_{i}, \widehat{v}_{i^{\prime}}, \widehat{v}_{j}, v_{-\left\{i, i^{\prime}, j\right\}}\right) \\
& \quad=x_{m}\left(\widehat{v}_{i}, \widehat{v}_{i^{\prime}}, \widehat{v}_{j}, v_{-\left\{i, i^{\prime}, j\right\}}\right)=x_{m+1}\left(\widehat{v}_{i}, \widehat{v}_{i^{\prime}}, \widehat{v}_{j}, v_{-\left\{i, i^{\prime}, j\right\}}\right)=\cdots=x_{n^{\prime}}\left(\widehat{v}_{i}, \widehat{v}_{i^{\prime}}, \widehat{v}_{j}, v_{-\left\{i, i^{\prime}, j\right\}}\right)=0 .
\end{aligned}
$$

In this manner, we can continue to apply Lemma 4(a) and 4(b) alternately. ${ }^{15}$ As the result of the application of Lemma 4(a) and 4(b), respectively, we find an agent with a valuation below $\bar{v}$ who is awarded an object, and an agent with a valuation above $\bar{v}$ who is not awarded an object. We apply again Lemma 4(b) and 4(a), respectively, to these two cases. The application of Lemma 4(a) will alternate the outcome of all agents with the valuation of $\bar{v}$ from not having an object allocated to having an object allocated. On the other hand, the application of Lemma 4(b) will alternate the outcome of them from having an object allocated to not having an object allocated. Notice that at each step there is one more agent with the valuation of $\bar{v}$ so that eventually there will be at least $m+1$ agents with valuation $\bar{v}$. This is a contradiction, since it is not possible to allocate more than $m$ objects. This will happen at the latest at $m$-times application. But depending on the number of agents who started with the valuation of $\bar{v}$, this could occur at an earlier step. Hence, efficiency must hold.

We have completed the proof of the Proposition.

15 Serizawa (2006), which is the previous version of this article, explains the details of this argument in the form of induction. 
Acknowledgments Ashlagi thanks Ron Lavi, Dov Monderer and Moshe Tennenholtz for helpful discussions. Serizawa is most thankful to Professor William Thomson for his detailed and helpful comments. Serizawa is also very thankful to the editor and two anonymous referee for reading the article thoroughly and their detailed comments. He benefitted from the discussion with the participants of the Eighth International Meeting of Social Choice and Welfare, July 2006 at Istanbul BİLGI University.

Open Access This article is distributed under the terms of the Creative Commons Attribution Noncommercial License which permits any noncommercial use, distribution, and reproduction in any medium, provided the original author(s) and source are credited.

\section{References}

Alkan A, Demange G, Gale D (1991) Fair allocation of indivisible goods and criteria of justice. Econometrica 59:1023-1039

Ashlagi I (July 2008) A characterization of anonymous truth-revealing position auctions. Harvard Business School, Harvard University, Discussion Paper

Atlamaz M, Yengin D (2008) Fair Groves mechanisms. Soc Choice Welf 31(4):573-587

Barberà S, Jackson M (1995) Strategy-proof exchange. Econometrica 63(1):51-87

Chew SH, Serizawa S (2007) Characterizing the Vickrey combinatorial auction by induction. Econ Theory 33(2):393-406

Green J, Laffont J-J (1977) Characterization of satisfactory mechanisms for the revelation of preferences for public goods. Econometrica 45:427-438

Groves T (1973) Incentives in teams. Econometrica 41:617-632

Hashimoto K, Saitoh H (2009) Strategy-proof and anonymous rule in queueing problems: a relationship between equity and efficiency. Osaka University, Mimeo

Holmström B (1979) Groves' scheme on restricted domain. Econometrica 47:1137-1144

Maskin (1987) On the fair allocation of indivisible goods. In: Feiwel G (ed) Arrow and the foundations of the theory of public policy. MacMillan, London, pp 342-349

Miyake M (1998) On the incentive properties of multi-item auctions. Int J Game Theory 27:1-19

Moulin H (1994) Serial cost sharing of excludable public goods. Rev Econ Stud 61:305-325

Moulin H (2010) Auctioning or assigning an object: some remarkable VCG mechanisms. Soc Choice Welf 34(2):193-216

Ohseto S (2006) Characterizations of strategy-proof and fair mechanisms for allocating indivisible goods. Econ Theory 29:111-121

Pápai S (2003) Groves' sealed bid auctions of heterogeneous objects with fair prices. Soc Choice Welf 20:371-386

Roth A, Sotomayor M (1990) Two-sided matching: a study in game-theoretic modeling and analysis, econometric society monographs. Cambridge University Press, Cambridge

Saitoh H, Serizawa S (2008) Vickrey allocation rule with income effect. Econ Theory 35(2):391-401

Sakai T (2008) Second price auctions on general preference domains: two characterizations. Econ Theory 37(2):347-356

Serizawa S (1999) Strategy-proof and symmetric social choice functions for public good economies. Econometrica $67(1): 121-145$

Serizawa S (March 2006) Strategy-proof and anonymous allocation rules of indivisible goods: a new characterization of Vickrey allocation rule. Institute of Social and Economic Research, Osaka University, Discussion Paper No. 648

Sprumont Y (1991) The division problem with single-peaked preferences: A characterization of the uniform allocation rule. Econometrica 59(2):509-519

Svensson LG (1983) Large indivisibles: an analysis with respect to price equilibrium and fairness. Econometrica 51:939-954

Tadenuma K, Thomson W (1991) No-envy and consistency in economies with indivisible goods. Econometrica 59:1755-1767

Vickrey W (1961) Counterspeculation, auctions, and competitive sealed tenders. J Finance 16(1):8-37

Walker M (1978) A note on the characterization of mechanisms for the revelation of preferences. Econometrica 46:147-152

Yengin D (2010) Egalitarian-equivalent Groves mechanisms in the allocation of heterogeneous objects. Soc Choice Welf (Forthcoming) 